\title{
Input-output Coupler System with 45-degree Slant Angle Based on Bragg Hologram
}

\author{
Anh-Hoang Phan, Nam Kim*, and Jae-Hyeung Park \\ Dept. of Computer and Communication, Chungbuk National University, Chungbuk 361-763, Korea \\ Kwon-Yeon Lee \\ Dept. of Electronic Engineering, Sunchon National University, Sunchon 540-742, Korea
}

(Received December 1, 2008 : revised January 92009 : accepted January 9 2009)

\begin{abstract}
In this paper, we designed and fabricated an input-output coupler system using two or three volume gratings at $632.8 \mathrm{~nm}$ wavelength. The additional third grating is added at the output coupler to enhance the overall efficiency. The experimental results show that the total system throughput reaches $63 \%$ with two-grating and $75 \%$ with three-grating input-output coupler. We also present a design method to obtain the desired output power ratio between the gratings.
\end{abstract}

Keywords: Holography, Gratings, Volume holographic gratings, Coupler

OCIS codes : (090.0090) Holography; (050.2770) Gratings; (050.7330) Volume holographic gratings; (060.1810) Couplers, switches, and multiplexers

\section{INTRODUCTION}

The input-output coupler based on holographic volume grating has been studied for several decades. Unlike the common prism coupler, the volume holographic grating coupler matches the fundamental requirement of the integrated optics. Therefore, many applications of a grating coupler have been explored in optical communications and optical data storage including optical data heads [1], optical transform filters, VLSI optical interconnections, optical memory, and multi-chip module clock-signal-distribution networks. Up to now, limited experiments have demonstrated a feasibility of the input output volume grating coupler. Coupling efficiencies of $62 \%$ for input at $633 \mathrm{~nm}$ laser [2], $23 \%$ for input and $5 \%$ for output at $850 \mathrm{~nm}$ laser [3] with a volume grating coupler have been achieved. M. L. Jones presented a method to simulate a coupler system with rectangular gratings in the normal direction [4]. However, these reports focus on the input coupler and output coupler efficiency separately not as a whole system or by simulation only. In this paper, we present a design and fabrication result of the coupler system at $632.8 \mathrm{~nm}$ wavelength

*Corresponding author: namkim@chungbuk.ac.kr with high efficiency. We analyze not only the individual grating efficiency but also the whole system throughput efficiency using rigorous coupled wave analysis (RCWA).

The gratings are recorded together on a sheet of photopolymer to make a system of couplers. At the output coupler, we insert one more grating to improve the power of the output beam. By this method, the output power is enhanced by $12 \%$ in comparison with output coupler of one grating. In this system, we define the total system throughput as the diffracted power from the output couplers divided by the power of the incident beam. In the experiment, the total system throughput of $63 \%$ with two-grating and $75 \%$ with three-grating inputoutput coupler were achieved. It is also possible to control the output beam power from each grating separately. We show that the desired output beam power ratio between the gratings can be obtained by adjusting the index modulation of each grating.

\section{DESIGN}

The basic configuration of the volume grating waveguide coupler is illustrated in Fig. 1(a). A free-space beam is incident on the waveguide at the designed angle. 


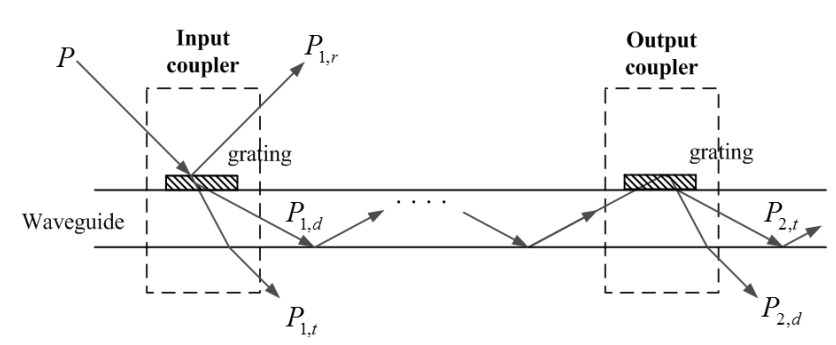

(a)

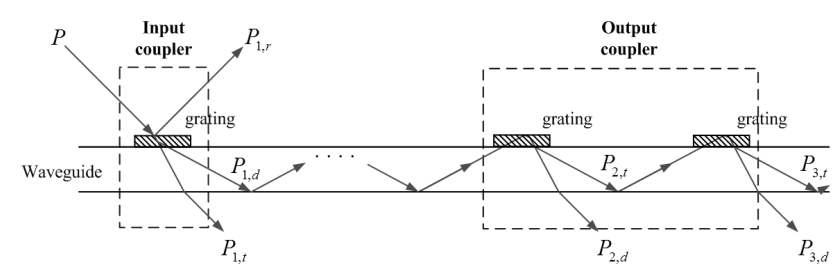

(b)

FIG. 1. Input-output coupler system: (a) Basic configuration, (b) Multi gratings configuration

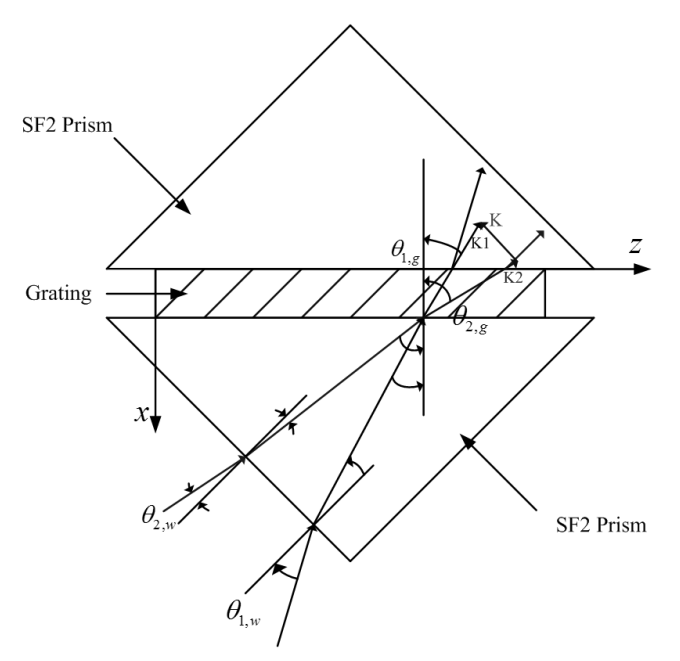

FIG. 2. Vector diagram for writing holographic Bragg gratings. SF2 prism is added to achieve desired recording angle.

This incident beam is diffracted to the waveguide modes by a volume hologram grating which is designed to satisfy the Bragg condition. This type of grating, therefore, is called a Bragg grating. The most remarkable advantage of using the Bragg grating is its capability of achieving higher diffraction efficiency. Because of the Bragg effect, it can be used to suppress multi-order diffraction and concentrate all diffracted light into a single diffraction order. As a result, theoretical diffraction efficiency near $100 \%$ can be reached [5]. Besides the basic configuration, we also simulated and fabricated a multi output grating configuration as shown in Fig. 1(b). The multi-grating configuration enhances the system efficiency as will be presented in the following. It is also useful when multi- path is required in the output stage.

In our system, the gratings are designed to meet the Bragg condition at $45^{\circ}$ incident-to-surface angle. Because of the simple grating profile, we used geometric analysis to calculate recording angles. The slant angle of the grating fringe planes was selected to be $45^{\circ}$ with respect to the surface normal.

The grating period $\Lambda$ was determined from the vector equation,

$$
\mathbf{K}=\mathbf{K}_{1}-\mathbf{K}_{2},
$$

where $\mathbf{K}$ is the grating vector, which has a magnitude of $2 \pi / \Lambda, \mathbf{K}_{1}$ and $\mathbf{K}_{2}$ are wave vectors of writing beams 1 and 2 as shown in Fig. 2. To meet the Bragg condition, $\Lambda$ must satisfy

$$
\begin{aligned}
& \Lambda=\lambda_{r} / 2 n_{g} \cos \left(\phi-\theta^{\prime}\right), \\
& \theta^{\prime}=\sin ^{-1}\left(\sin \theta / n_{g}\right),
\end{aligned}
$$

where $\lambda_{\mathrm{r}}$ is the reading beam wavelength, $n_{\mathrm{g}}$ is the average refractive index of the grating, $\phi$ is grating slant angle, $\theta$ is incident angle, and $\theta^{\prime}$ is the refraction angle inside the grating region. With parameters $n_{\mathrm{g}}=1.5$ and $\phi=45^{\circ}$, we have grating period $\Lambda$ equal to $726.66 \mathrm{~nm}$ at $632.8 \mathrm{~nm}$ reading wavelength. Decomposing $\mathbf{K}, \mathbf{K}_{1}$, and $\mathbf{K}_{2}$ into their and $z$ axes, we get

$$
\begin{aligned}
& K_{x}=K_{1 x}-K_{2 x} \\
& K_{z}=K_{1 z}-K_{2 z}, \\
& K_{1 x}^{2}+K_{1 z}^{2}=K_{0}^{2} n_{g}^{2} \\
& K_{2 x}^{2}+K_{2 z}^{2}=K_{0}^{2} n_{g}^{2}
\end{aligned}
$$

where $k_{0}$ is the wave number of the writing beam in free space. Using Eq. (4) and the design value of $\mathbf{K}$ we can determine $K_{1 x}, K_{1 z}, K_{2 x}$, and $K_{2 z}$. From these wave vectors, the incidence angles of the recording beams in the grating are $\theta_{1, \mathrm{~g}}=\arctan \left(K_{1 z} / K_{1 x}\right)$ and $\theta_{2, g}=\arctan$ $\left(K_{2 z} / K_{2 x}\right)$. Note that the shrinkage of the recording medium should be considered in the calculation of Eq. (4). The photopolymer DuPont HRF-600, which we used as a recording medium in our system, exhibits the shrinkage of $\delta$ during the exposure and the curing process. In general, the $K_{z}$ component is not affected by the shrinkage because of its large dimension. However, the $K_{x}$ component is affected, after exposure and curing, $K_{x}$ is given by $K_{x}=K_{x, \text { orig }}(1-\delta)$, where $K_{x, \text { orig }}$ is the originally recorded $K_{x}$ component. Therefore, pre-compensation is needed for this shrinkage. This is accomplished by recording compensated $K_{x, \text { orig }}$ to obtain the desired $K_{x}$.

The model of the shrinkage effect is shown in Fig. 3. We can see that the grating period $\Lambda$ and the slant 


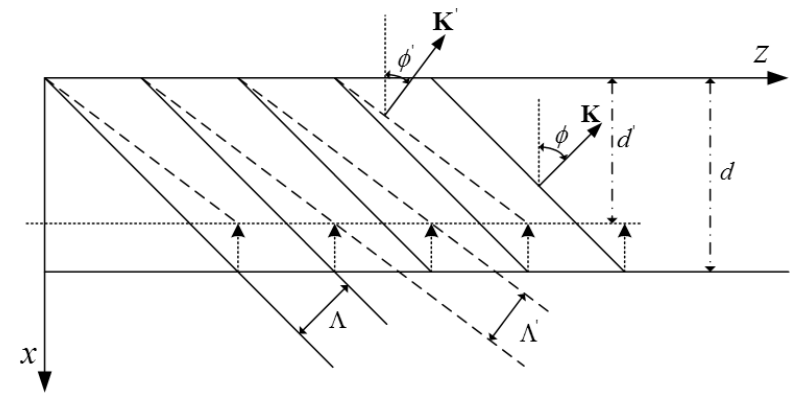

FIG. 3. Fringe-plane rotation model of the shrinkage effect. The initial thickness reduces to after shrinkage.

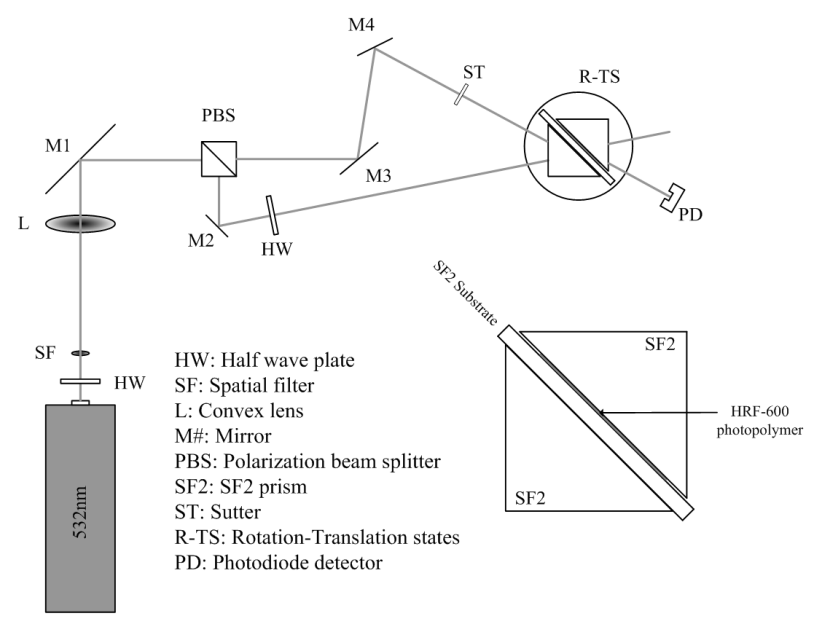

FIG. 4. Experimental setup

angle $\phi$ change because of the shrinkage effect. The new grating period $\Lambda^{\prime}$ and the slant angle $\phi^{\prime}$ at the shrinkage factor $\delta$ are given by

$$
\begin{aligned}
& \Lambda^{\prime}=\left|\frac{\sin \phi^{\prime}}{\sin \phi}\right| \Lambda \\
& \tan \left(\phi^{\prime}\right)=(1-\delta) \tan (\phi)
\end{aligned}
$$

A SF2 ${ }^{1)}$ prism is added in front of the sample in order to achieve desired $\theta_{1, \mathrm{~g}}$ and $\theta_{2, \mathrm{~g}}$ as shown in Fig. 2 . Finally, another prism is placed behind the sample in order to minimize reflections from the sample-air interface that could affect the pattern being recorded [6]. SF2 prism and DuPont HRF-600 indexes are 1.6457 and 1.51 respectively (at $532 \mathrm{~nm}$ laser). Using Snell's law we have writing angles of $\theta_{1, w}=28.4^{\circ}, \theta_{2, w}=11.4^{\circ}$ for shrinkage $\delta=0$.

\section{EXPERIMENTAL SETUP}

In the experiment, we record all gratings on one photo-

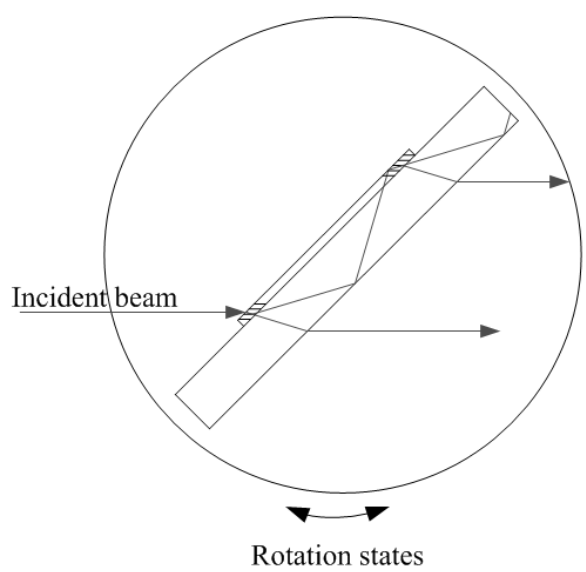

(a)

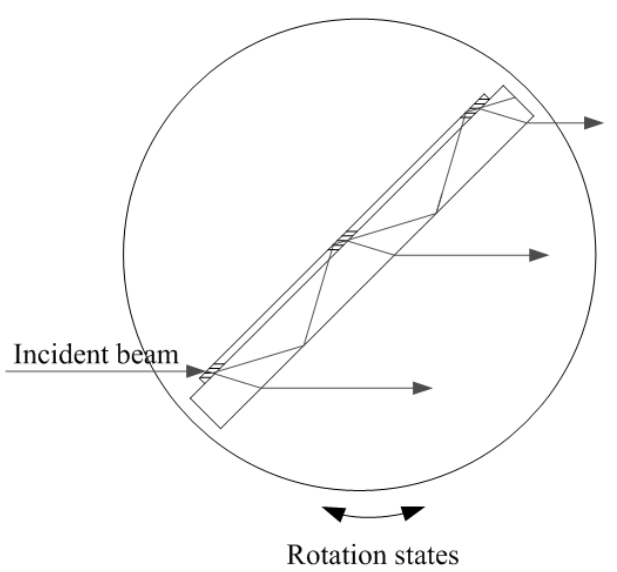

(b)

FIG. 5. Diagram of testing configuration: (a) Two-grating system, (b) Three-grating system

polymer layer. Fig. 4 shows the experimental setup to fabricate the input-output coupler system. The writing wavelength is $532 \mathrm{~nm}$ Nd:YAG laser light source. The recording beam 1 and recording beam 2 go to the sample under the recording angles. The sample is placed on a SF2 glass substrate and held by a couple of SF2 prisms. SF2 matching oil is used to connect adjacent layers. The system of sample, substrate and prisms are placed on a rotation stage which is on top of the three-dimensional (3-D) translation stages. Therefore, we can control these systems to record one, two, or three gratings on a photopolymer sheet to obtain an input-output coupler system with the same recording conditions.

Fig. 5 shows the configuration for measuring the total systems throughput. The grating is placed on a rotation stage with a remote control panel to control the incident beam angle. Two or three photo-detectors are used separately to measure the output beam power of each grating.

1) The "SF" abbreviation stands for "Schwerflint," that is, "heavy flint" in German. It normally called "dense flint". 


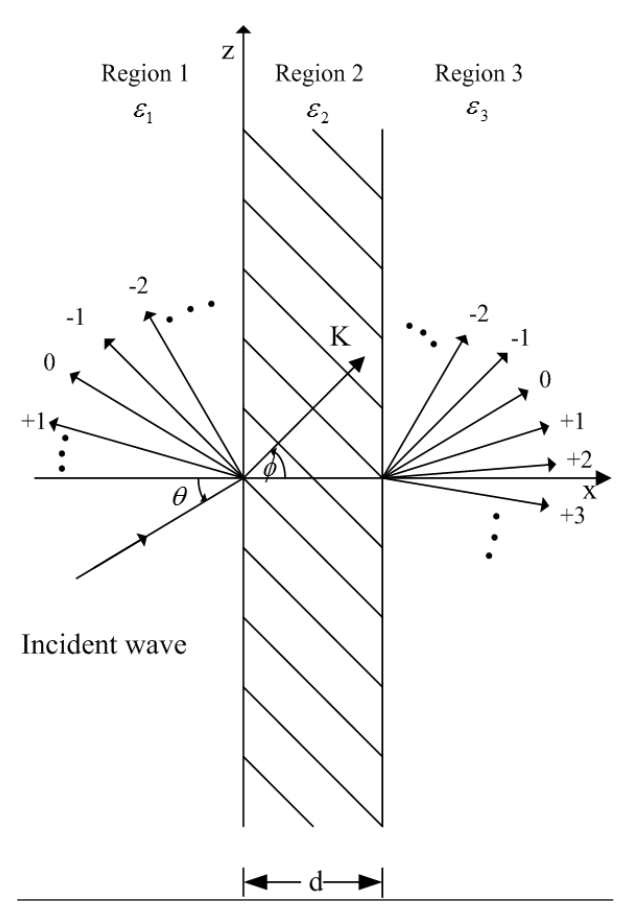

FIG. 6. Diffraction from planar grating

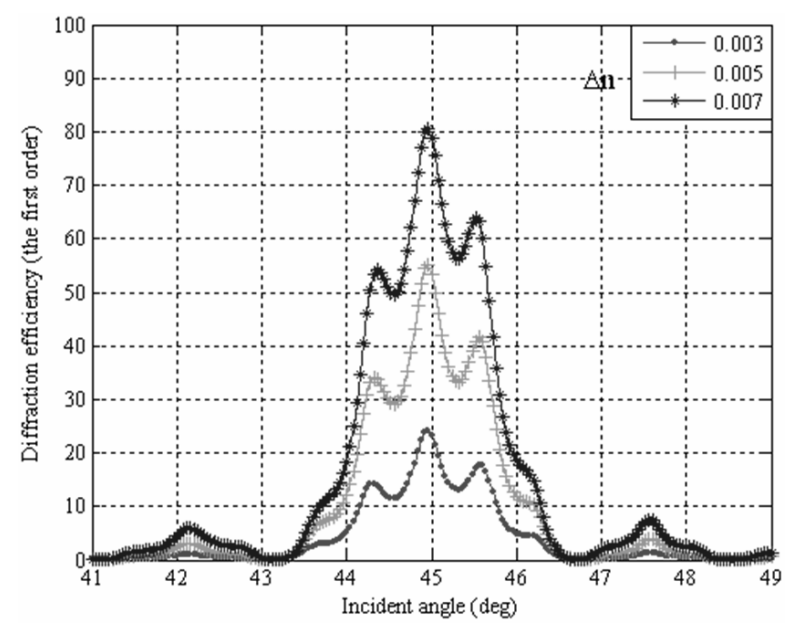

FIG. 7. Diffraction efficiency

\section{SIMULATION AND EXPERIMENT RESULT}

Input and output coupler efficiencies have been extensively studied. Several different techniques have been used to analyze the diffraction of electromagnetic waves by the spatially modulated media. Among them, one of the most useful methods is the RCWA, which has an advantage in analyzing electromagnetic waves such that it provides high accuracy without any approximations.

RCWA of planar-grating diffraction has been reported by M.G. Moharam and T.K. Gaylord [7]. The geometry of diffraction from the planar grating is shown in Fig. 6 . The incident beam from air (Region 1) goes to the Bragg
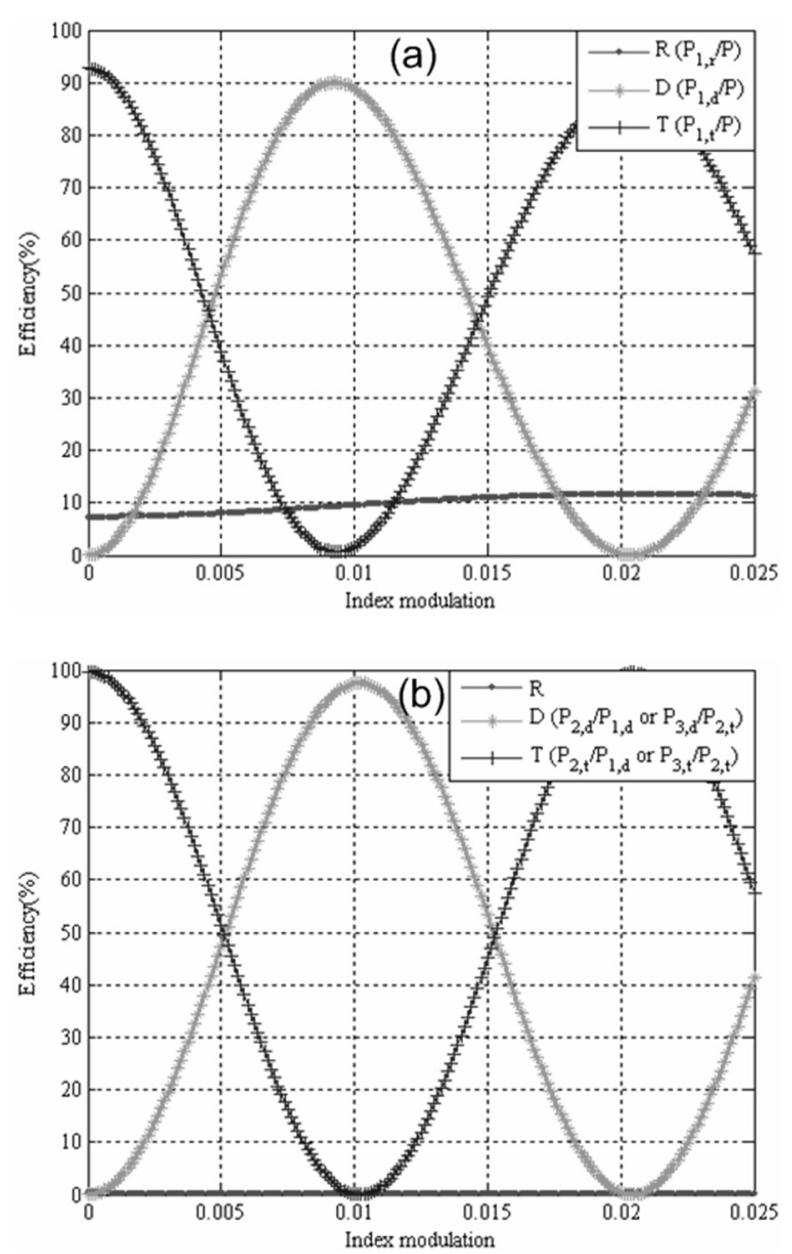

FIG. 8. Theoretical efficiencies versus index modulation - $\Delta n$ : (a) The first grating, (b) The second and/or third grating.

grating (Region 2) and is diffracted to the SF2 glass substrate (Region 3). The incident beam goes to the planar-grating and is then reflected and diffracted in many directions, i.e. orders. Following Moharam and Gaylord's method, we get the relation between reflection efficiency $\mathrm{R}$, transmission efficiency $\mathrm{T}$, diffraction efficiency $\mathrm{D}$, index modulation and the incident angle.

We applied Moharam and Gaylord's method to our input-output coupler system. Fig. 7 shows the diffraction efficiency variation of one grating with two important simulation conditions: incident angle and index-modulation. Diffraction efficiency goes to the maximum point when the incident angle meets the Bragg condition. It is also observed that the larger index modulation enhances the diffraction efficiency. The relationship between $\mathrm{R}, \mathrm{T}$, $\mathrm{D}$ and index modulation at the Bragg angle is shown clearly in Fig. 8. At the first grating, the incident beam comes from the air to the photopolymer plate. This beam will lose about $10 \%$ of power because of reflection (Fig. $8(\mathrm{a}))$. At the other gratings, the incident beam comes from the same medium, so it will be totally reflected 

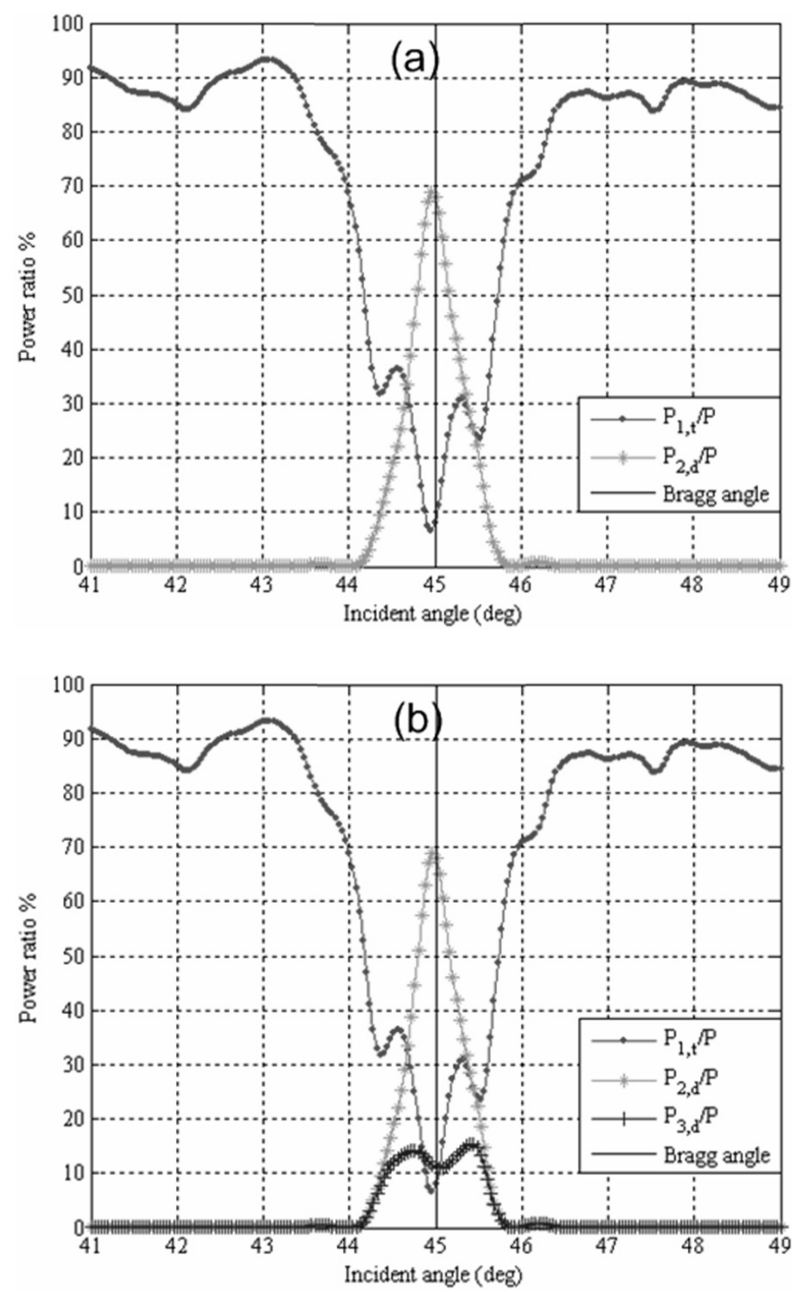

FIG. 9. Simulation results for input-output coupler systems with index modulation is 0.007: (a) Two-grating system, (b) three-grating system

at the boundary (Fig. 8(b)). Therefore, unlike the Kogelnik's method [8] if we ignore absorption loss, the efficiency of all systems can reach $90 \%$ in theory.

Fig. 9 is the simulation results for the output power of the proposed coupler system. The output power efficiency of the two-grating system is shown in Fig. 9(a) while the three-grating system is shown in Fig. 9(b). Three gratings are assumed to be recorded with the index modulation 0.0075 , which is achievable in practical experiments. We see that the total system throughput reaches $68 \%$ for two-gratings and is enhanced by $12 \%$ in case of three-gratings. Hence, the total system throughputs $T T_{2 \mathrm{~g}}$ for two grating and $T T_{3 g}$ for three grating coupler are given as

$$
T T_{2 g}=\frac{P_{2, d}}{P}, T T_{3 g}=\frac{P_{2, d}+P_{3, d}}{P}
$$

where $P$ is the incident power, $P_{2, d}$ is the diffracted power from the second grating and $P_{3, d}$ is the diffracted power from the third grating as shown Fig. 1.

Generally, it is desirable to maximize the output beam power $P_{2, d}$ and $P_{3, d}$. In some applications, however, it might be required to maintain the desired ratio between the output beam powers. The proposed coupler system can be made with a desired ratio of the output power. Assume that the power ratio transmitted through three gratings is given as

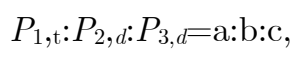

where $P_{1, \mathrm{t}}$ is the transmitted power from the first grating (Fig. 1). The output power for each grating is calculated by

$$
\begin{aligned}
& P_{1, t}=a \times \frac{P-P_{\text {loss }}-P_{3, t}}{a+b+c} \\
& P_{2, d}=b \times \frac{P-P_{\text {loss }}-P_{3, t}}{a+b+c}, \\
& P_{3, d}=b \times \frac{P-P_{\text {loss }}-P_{3, t}}{a+b+c}
\end{aligned}
$$

where $P_{\text {loss }}$ is the power loss of the system and $P_{3, t}$ is the power transmitted through the third grating, which is negligible when the efficiency of the third grating is nearly $100 \%$. In theory, we can get a coupler system with any ratio between the output powers from Eq. (9). As mentioned before, we know that the main loss is the reflection loss at the air-grating interface of the input grating. Therefore, the power loss is approximated by

$$
P_{\text {loss }}=10 \% \times P
$$

Eqs. (9) and (10) give us a formula to get the desired output power for each grating with the desired ratio. From Fig. 8, we can estimate the index modulation value for desired diffraction efficiency or transmission efficiency. For an example, the ratio is 1:1:1. Firstly, we calculate the output power from each grating.

$$
\begin{aligned}
& P_{1, t}=30 \% \times P \\
& P_{1, d}=30 \% \times P \\
& P_{1, t}=30 \% \times P
\end{aligned}
$$

Eq. (11) shows that the transmitted power from the first grating, the diffracted power from the second and the diffracted power from third grating are $1 / 3$ of the incident power. Hence, the transmission efficiency of the first grating $P_{1, t} / P$ is $30 \%$; the diffraction efficiency of the second grating $P_{2, d} / P_{1, d}$ is $50 \%$; the diffraction efficiency of the third grating $P_{3, d} / P_{2, t}$ is nearly $100 \%$. After that, Fig. 8 is used to estimate the required index modulation for each grating. The index modulation for the 

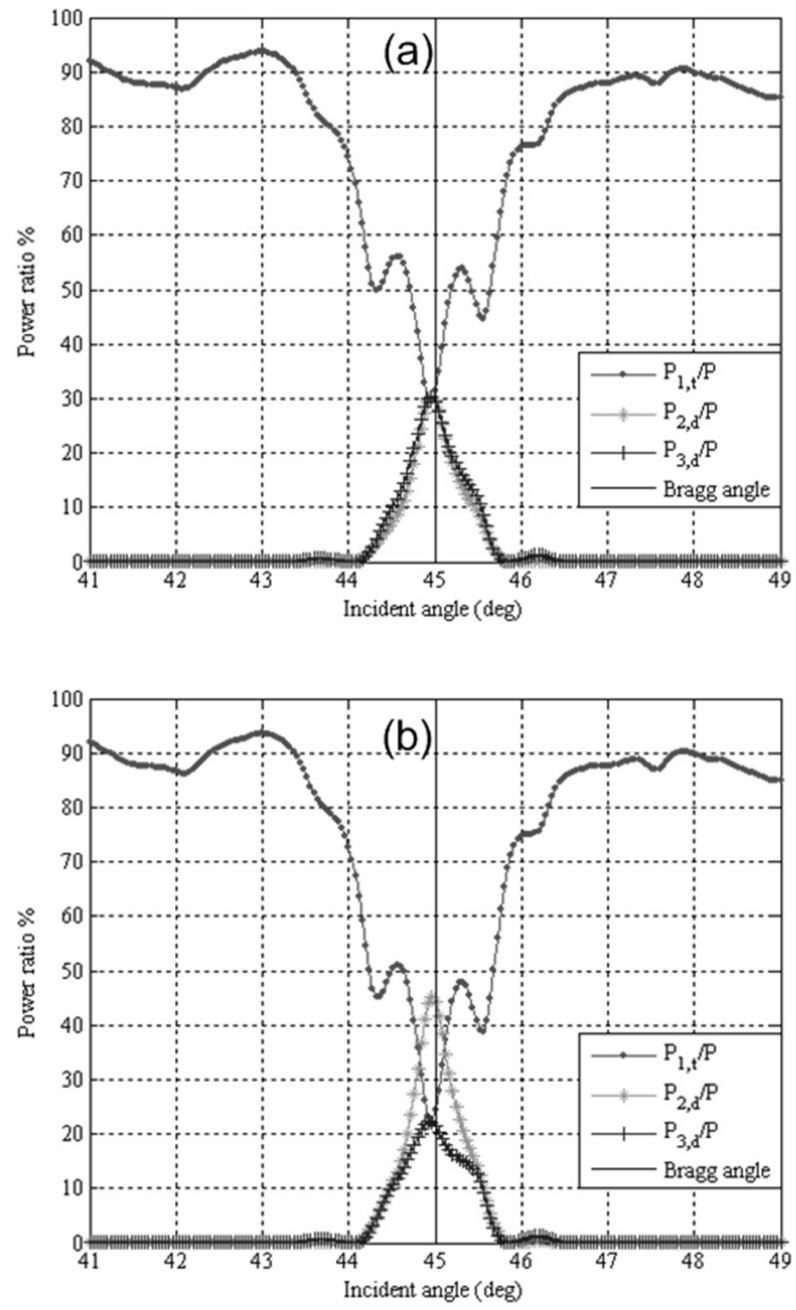

FIG. 10. Simulation result for fixed ratio power: (a) Ratio power is $1: 1: 1$, (b) Ratio power is $1: 2: 1$

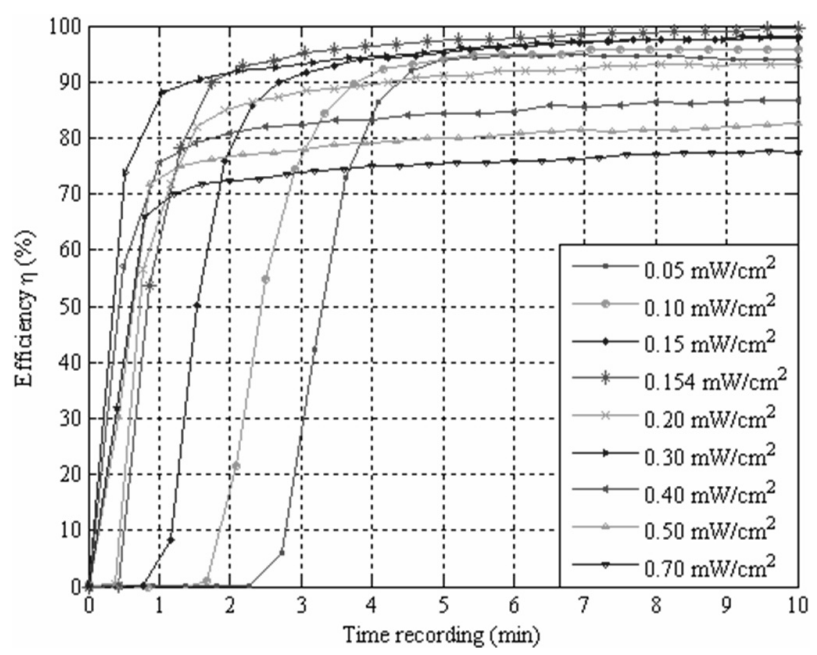

FIG. 11. Experimental result: Optical property of photopolymer according different exposure energy
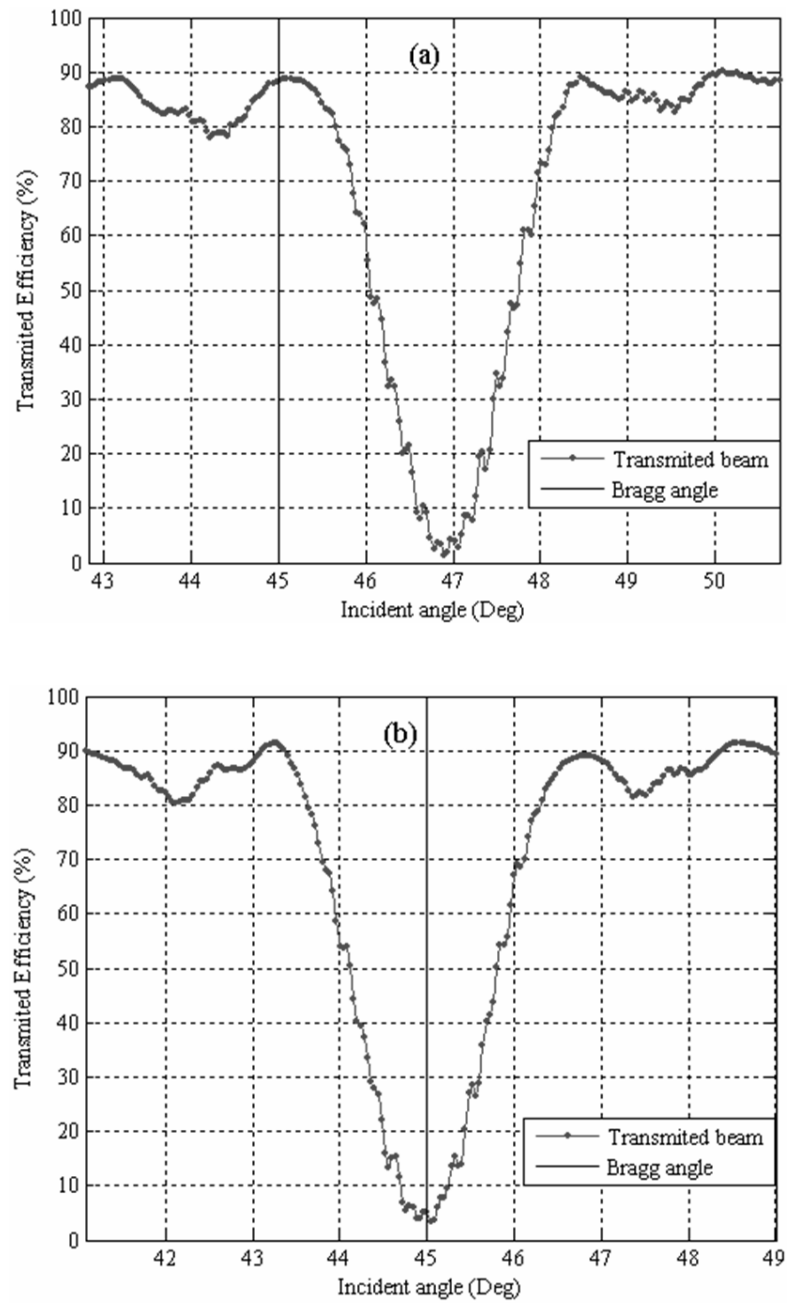

FIG. 12. Experimental result: Transmitted efficiency: (a) Recording without any consideration of the shrinkage, (b) Recording with $4.76 \%$ shrinkage

first grating is 0.0055 , for the second grating is 0.0051 , and for the third grating is 0.010. Fig. 10(a) illustrated the output power for 1:1:1 ratio. Fig. 10(b) shows the output power ratio 1:2:1 which is obtained under the same procedure.

We experimentally fabricated two and three grating waveguides. Firstly, the characteristic of the photopolymer DuPont HRF-600 was investigated. We recorded a pure grating (non slant grating) with the recording angle of $15^{\circ}$ for each recording beam which is TE polarization. Fig. 11 shows how sensitive DuPont HRF-600 photopolymer is to the intensity of the writing beams. It can be observed that with $0.154 \mathrm{~mW} / \mathrm{cm}^{2}$, we get the maximum diffracted efficiency.

In order to measure the shrinkage of the photopolymer, at the first recording we record one grating without any consideration of the shrinkage. After recording, we fix the photopolymer with a UV light source for ten minutes and then clean with $95 \%$ alcohol. The transmitted beam 


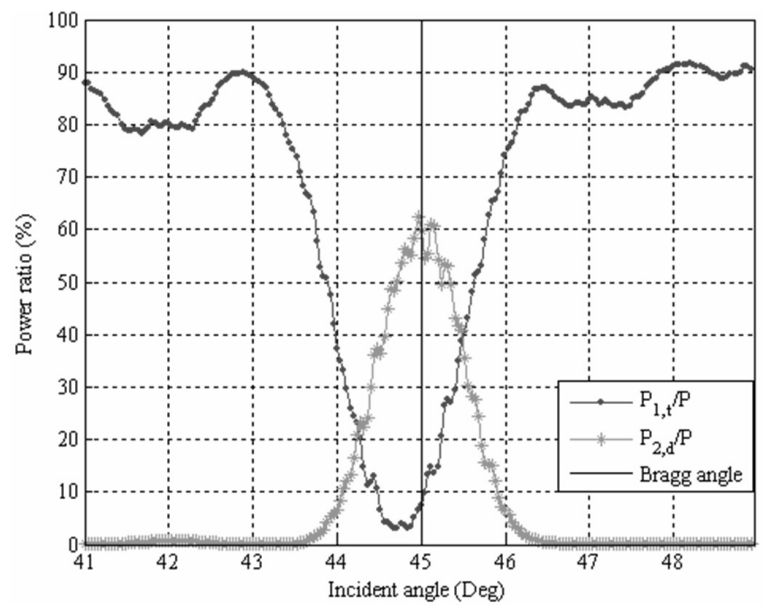

FIG. 13. Experimental result: Two gratings input-output coupler output-power

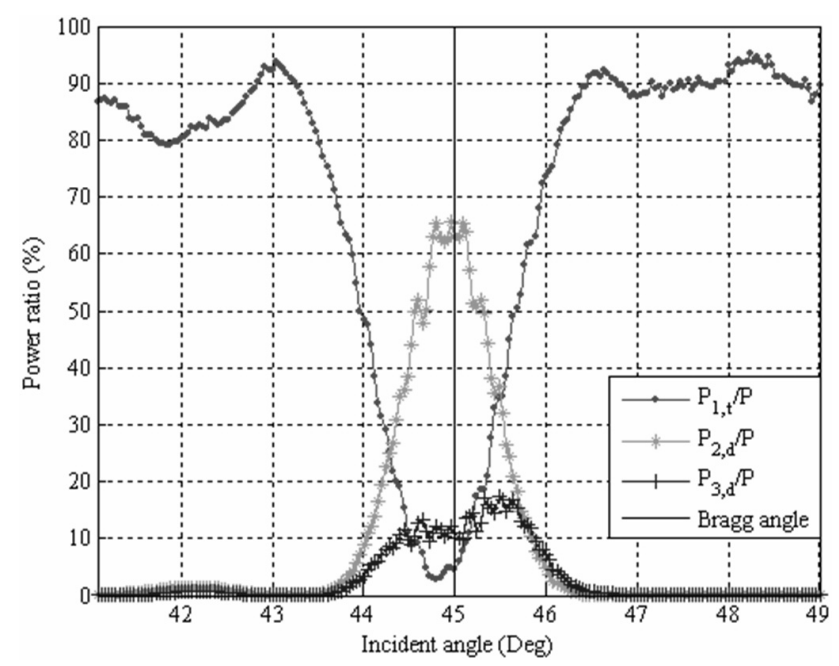

FIG. 14. Experimental result: Three gratings input-output coupler output-power

efficiency versus the incident angle of this recording is plotted in Fig. 12(a). The shrinkage factor is calculated by using Eqs. (2), (5), (6), and the angle at minimum point of transmitted power. The shrinkage factor is estimated to $4.76 \%$ after fixing and cleaning process. The transmission efficiency of the grating after compensating $4.76 \%$ shrinkage is shown in Fig. 12(b). The Bragg condition is now satisfied at this recorder.

Fig. 13 and 14 show the experimental results for two and three grating waveguides. We reached $63 \%$ systems throughput for two grating coupler systems and more than $75 \%$ for three gratings coupler systems. Because all the gratings are recorded on one sheet of photopolymer sequentially, each grating has a slight difference in shrinkage factor. This difference makes a small shift of transmission beam from the Bragg angle as we see in Fig. 13 and 14. It also shows that the transmitted beams and diffracted beams from the first grating have two small peaks beside the main peak; it is slightly different with simulation results, which have two significant peaks beside the main peak. But the shape for diffracted beam from the second and third grating is the same as for the simulation results.

\section{CONCLUSION}

In this research, we fabricated a coupler system using holographic Bragg gratings for $632.8 \mathrm{~nm}$ wavelength with high efficiency. The efficiency is examined in terms of the whole system throughput not restricted to the input efficiency or the output efficiency separately. An additional output grating makes better use of the input beam, enhancing the system throughput more than $10 \%$. In addition, the simulation results also show that the power ratio between the output beams can be controlled by adjusting the index-modulation of each grating. Hence, we can choose to get the maximum power at the output coupler or the desired ratio between each of output beams. In the experiment, we designed the system to get the maximum output power. The total system throughput reached $63 \%$ for two gratings and $75 \%$ for three gratings. The simulation and experiment results show that the total system throughput is enhanced with the increase of index modulation, and degraded quickly as the incident angle deviates from the Bragg angle. The full width at half maximum is about 1 degree. Hence it is convenient for adjustment in practical application.

\section{ACKNOWLEDGMENT}

This work was supported by the Korea Research Foundation Grant funded by the Korean Government (Ministry of Education, Science and Technology) (The Regional Research Universities Program/ Chungbuk BIT Research-Oriented University Consortium)

\section{REFERENCES}

1. C. H. Shin et al., "Holographic polarization-selective module based on a small Dove prism coupler for magnetooptical pickup heads," Appl. Opt. 44, 4248-4254 (2005).

2. W. Driemeier, "Bragg-effect grating couplers integrated in multi-component polymeric waveguides," Opt. Lett. 15, 725-727 (1990).

3. Q. Huang and R. R. Ashley, "Holographic Bragg grating input-output couplers for polymer waveguides at an 850-nm wavelength,” Appl. Opt. 36, 1198-1203 (1997).

4. M. L. Jones, R. P. Kenan, and C. M. Verber, "Rectangular characteristic gratings for waveguide input and output coupling," Appl. Opt. 34, 4149-4158 (1995).

5. J. W. Goodman, Introduction to Fourier Optics (McGraw- 
Hill, New York, 1996).

6. R. A. Villalaz, "Volume grating couplers for optical interconnects: analysis, design, fabrication and test," (Georgia Institute of Technology, Thesis, 2004).
7. M. G. Moharam and T. K. Gaylord, "Rigorous coupledwave analysis of planar-grating diffraction," J. Opt. Soc. Am 71, 811-818 (1981).

8. H. Kogelnik, "Coupled wave theory for thick hologram gratings,” Bell Syst. Tech. J. 48, 2909-2947 (1969). 